\title{
Synbiotic supplementation and the effects on clinical and metabolic responses in patients with rheumatoid arthritis: a randomised, double-blind, placebo-controlled trial
}

\author{
Batol Zamani ${ }^{1}$, Shima Farshbaf ${ }^{2}$, Hamid R. Golkar ${ }^{1}$, Fereshteh Bahmani ${ }^{2}$ and Zatollah Asemi ${ }^{2 *}$ \\ ${ }^{1}$ Department of Gastroenterology, Kashan University of Medical Sciences, Kashan PO Box 8715988141, Iran \\ ${ }^{2}$ Research Center for Biochemistry and Nutrition in Metabolic Diseases, Kashan University of Medical Sciences, \\ Kashan PO Box 8715988141, Iran \\ (Submitted 9 February 2017 - Final revision received 10 March 2017 - Accepted 14 March 2017 - First published online 11 May 2017)
}

\section{Abstract}

Synbiotic intake may be associated with reduced inflammation in patients with rheumatoid arthritis (RA) due to optimised inflammatory markers, oxidative stress and insulin resistance. This research was conducted to assess the effects of synbiotic supplementation on the clinical and metabolic parameters of patients with RA. A total of fifty-four patients with RA were allocated into two groups to receive either a synbiotic capsule $(n 27)$ or a placebo $(n 27)$ for 8 weeks in this randomised, double-blind, placebo-controlled trial. Fasting blood samples were taken at baseline and week 8 of the study to quantify related markers. After the 8-week intervention, compared with the placebo, synbiotic supplementation resulted in a significant reduction in serum high-sensitivity C-reactive protein (hs-CRP) levels $(-1427 \cdot 8$ (SD 3267.2) $v .+2833 \cdot 4($ SD $5639 \cdot 7) \mathrm{ng} / \mathrm{ml}, P=0 \cdot 001)$. In addition, compared with the placebo, synbiotic supplementation improved disease activity score-28 joints (DAS-28) ( $-1 \cdot 6$ (SD $0 \cdot 8) v$. $-0 \cdot 3$ (SD $0 \cdot 5$ ), $P<0.001)$ and visual analogue scales (VAS) pain ( -30.4 (sD 18.7) $v .-11.5$ (sD 15.9), $P<0.001$ ). In addition, a significant elevation in plasma nitric oxide (NO) (+0.8 (sD 4.4) $v \cdot-2 \cdot 6$ (sD 4.5) $\mu \mathrm{mol} / \mathrm{l}, P=0 \cdot 008$ ), and significant reductions in insulin values $(-13 \cdot 8$ (sD 26.4) $v \cdot+4 \cdot 2$ (sD $28 \cdot 2) \mathrm{pmol} / \mathrm{l}$, $P=0 \cdot 01$ ), homoeostasis model of assessment-estimated insulin resistance (HOMA-IR) (-0.5 (SD 1.0) $v .+0 \cdot 1$ (sD $1 \cdot 1$ ), $P=0 \cdot 03)$ and homoeostatic model assessment- $\beta$-cell function (HOMA-B) (-9.4 (sD 17.9) $v .+3 \cdot 3$ (sD 18.9), $P=0.01$ ) following supplementation with the synbiotic compared with the placebo. Compared with the placebo, synbiotic supplementation also resulted in a significant increase in plasma GSH (+36.6 (SD 63.5) v. -58.5 (sD 154.4) $\mu \mathrm{mol} / \mathrm{l}, P=0.005$ ). Overall, our study demonstrated that synbiotic supplementation for 8 weeks among patients with RA had beneficial effects on hs-CRP, DAS-28, VAS, NO, insulin levels, HOMA-IR, HOMA-B and GSH levels.

Key words: Synbiotics: Supplementation: Rheumatoid arthritis: Metabolic profiles

Rheumatoid arthritis (RA) is an imperative, chronic, autoimmune and inflammatory disease of indistinct origin with its greatest impact on the joints of the body ${ }^{(1)}$. Recent evidence suggests that subjects with RA have significant changes in intestinal microbiota compared with healthy subjects ${ }^{(2)}$. In addition, RA subjects show a significant reduction in the quantity of Bifidobacterium species and lactic acid bacteria ${ }^{(3)}$. Average homoeostatic model of assessment for insulin resistance (HOMA-IR) levels in patients with RA were reported to be $31 \%$ higher than that in the healthy population ${ }^{(4)}$. Impaired insulin metabolism, increased indices of inflammation and oxidative stress play a significant role in the pathogenesis of $\mathrm{RA}^{(5-7)}$, which in turn would result in an increased risk of fatal cardiovascular events by $50 \%{ }^{(8)}$ and type 2 diabetes mellitus (T2DM) by $68 \%$ in men and $46 \%$ in women ${ }^{(9)}$.
Few studies have previously evaluated the effects of probiotic supplementation on clinical and metabolic parameters in RA subjects with conflicting findings. Our study among RA subjects has shown that probiotic administration for 8 weeks had beneficial effects on clinical symptoms, serum insulin and high-sensitivity C-reactive protein (hs-CRP) values, but did not influence insulin resistance and sensitivity, lipid profiles and other parameters of inflammation and oxidative stress ${ }^{(10)}$. Furthermore, in an animal study, intake of probiotic Bacillus coagulans plus prebiotic inulin significantly improved the biochemical and clinical parameters of induced $\mathrm{RA}^{(3)}$. Likewise, few animal studies in RA have demonstrated that treatment with probiotics was associated with decreased arthritic severity through reduced gut permeability ${ }^{(11,12)}$. Synbiotics refer to nutritional supplements combining probiotics and prebiotics in

Abbreviations: DAS-28, disease activity score-28 joints; HOMA-B, homoeostatic model assessment- $\beta$-cell function; HOMA-IR, homoeostasis model of assessment-estimated insulin resistance; hs-CRP, high-sensitivity C-reactive protein; KUMS, Kashan University of Medical Sciences; MDA, malondialdehyde; RA, rheumatoid arthritis; TAC, antioxidant capacity; VAS, visual analogue scales.

* Corresponding author: Z. Asemi, fax +98 315546 3377, email asemi_r@yahoo.com 
a form of synergism ${ }^{(13)}$. Previous studies have demonstrated that the synergistic effects of synbiotic supplementation on the intestinal and faecal microflora and immune system are significantly greater than the effects of either prebiotic or probiotic supplementation alone ${ }^{(14,15)}$. Synbiotic supplementation for 8 weeks among patients with T2DM also decreased inflammatory factors ${ }^{(16)}$. In addition, synbiotic supplementation for 28 weeks among patients with the metabolic syndrome resulted in statistically significant improvements in insulin resistance indices, TAG, total- and HDL-cholesterol levels, whereas LDL-cholesterol levels remained unchanged ${ }^{(17)}$.

Synbiotic supplementation might improve glucose metabolism, lipid profiles and inflammatory factors through the modification of gut flora, the reduction of endotoxin levels, elevation of faecal $\mathrm{pH}$ via the production of $\mathrm{SCFA}^{(18)}$ and the reduction of pro-inflammatory cytokine production ${ }^{(19)}$. Given the anti-inflammatory effects of synbiotics, we hypothesised that synbiotic supplementation might help RA patients to control their clinical signs, biomarkers of inflammation and oxidative stress, and insulin resistance. This research was, therefore, performed to determine the effects of synbiotic supplementation on clinical and metabolic parameters in patients with RA.

\section{Methods \\ Participants}

This study, registered in the Iranian website (www.irct.ir) for registration of clinical trials (http://www.irct.ir: IRCT201611 165623N94), was a randomised double-blind clinical trial that was conducted among fifty-four patients with RA referred to the Shahid Beheshti Hospital in Kashan, Iran, according to the 1987 American College of Rheumatology criteria $^{(20)}$, diagnosed at least 6 months ago with moderate and severe disease activity (disease activity score-28 joints: DAS-28 $>3 \cdot 2$ ), aged $25-70$ years from September 2016 to December 2016. Disease activity was evaluated on the basis of DAS-28 ${ }^{(21)}$. Patients who have chronic renal failure, pregnant or lactating women, symptoms or personal history of CVD, diabetes mellitus, the consumption of antihyperglycaemic agents including metformin, patients unlikely to come for follow-up in the following 3 months and patients who are unable to read numbers and/or unable to mark the pain scale, taking probiotics, synbiotics, antioxidant and/or anti-inflammatory supplements such as vitamin E, vitamin $\mathrm{C}$ and $n-3$, and taking antibiotics were excluded from the study. The study protocol was approved by the research ethics committee of Kashan University of Medical Sciences (KUMS) (reference no. IR.Kaums.REC.1395.46) and informed consent was obtained from all subjects.

\section{Study design}

At first, participants were randomised into two groups for the intake of either synbiotic supplements ( $n$ 27) or the placebo $(n 27)$ for 8 weeks. In the treatment group, participants received a synbiotic capsule containing Lactobacillus acidophilus, Lactobacillus casei and Bifidobacterium bifidum $\left(2 \times 10^{9}\right.$ colonyforming units/g each) plus $800 \mathrm{mg}$ inulin. Because of the lack of evidence about the appropriate dosage of probiotics and inulin for RA patients, we used the above-mentioned doses of probiotics and inulin based on observed beneficial effects on markers of insulin metabolism in gestational diabetes (GDM) women $^{(22)}$ and observed beneficial effects of probiotics on hs-CRP in patients with RA ${ }^{(10)}$. In the current study, the duration of the intervention was selected on the basis of the observed beneficial effects of probiotic supplementation on inflammatory factors in patients with $\mathrm{RA}^{(10,23)}$. Participants in the placebo group received a placebo containing starch but no bacteria. The appearance of the placebo was indistinguishable in colour, shape, size, packaging, smell and taste from the synbiotic capsule. Synbiotic supplements and placebos were produced by Tak Gen Zist Pharmaceutical Company. Quality control of synbiotic supplements was conducted in the laboratory of the Food and Drug Administration in Tehran. Randomisation assignment was performed using computer-generated random numbers. Randomisation and allocation were concealed from the researchers and patients until the final analyses were completed. The randomised allocation sequence, enrollment of patients and allocation to interventions were conducted by trained staff. Patients were requested not to change their routine physical activity, and not to take any supplements that might affect their nutritional status during the 8-week treatment. A 3-d food record and physical activity records were completed by all participants at baseline and at weeks 2, 5 and 8 of intervention. The dietary records were based on estimated values in household measurements. To obtain macronutrient and micronutrient intakes of participants based on these 3-d food diaries, we used Nutritionist IV software (First Databank) modified for Iranian foods. Physical activity was described as metabolic equivalents $(\operatorname{MET})(\mathrm{h} / \mathrm{d})^{(24)}$. To determine the MET for each patient, we multiplied the time $(\mathrm{h} / \mathrm{d})$ reported for each physical activity by its related MET coefficient using standard tables ${ }^{(24)}$. Compliance with the synbiotic intake was evaluated by asking patients to bring the medication containers.

\section{Assessment of anthropometric measurements}

Weight and height (Seca) were determined before and after the 8-week treatment in a fasting state, without shoes and in minimal clothing, by a trained staff member. BMI was calculated using the height and weight measurements (weight $\left.(\mathrm{kg}) /(\text { height }(\mathrm{m}))^{2}\right)$.

\section{Assessment of outcomes}

The primary outcome end-points were inflammatory factors and DAS-28 in the current study. The secondary outcome endpoints were insulin metabolism, lipid concentrations and biomarkers of oxidative stress.

\section{Clinical assessment}

At baseline and after the 8-week intervention, we collected data on: the number of tender and swollen joints on the basis of the twenty-eight-joint count, visual analogue scales (VAS) (0-100 mm) for pain and DAS-28. All clinical assessments were conducted blindly by a single experienced clinician. 


\section{Biochemical assessment}

The 12-h fasting blood samples were obtained from participants at baseline and at week 8 of the treatment at the Kashan reference laboratory. The samples were stored at $-80^{\circ} \mathrm{C}$ until analysis at the KUMS reference laboratory. Serum hs-CRP values were quantified by the use of a commercial ELISA kit (LDN) with a limit of detection (LoD) of $10 \mathrm{ng} / \mathrm{ml}$, and with intra- and interassay CV 3.7 and $5.6 \%$, respectively. Plasma nitric oxide (NO) was determined by the Griess method ${ }^{(25)}$. To quantify fasting plasma glucose (FPG), serum TAG, VLDL-, total-, LDL- and HDLcholesterol values, we used available kits (Pars Azmun) with inter- and intra-assay CV $<5 \%$. Serum insulin was assessed using an ELISA kit (Monobind) with an LoD of $0 \cdot 114 \mu \mathrm{IU} / \mathrm{ml}$, and the intra- and inter-assay CV 3.0 and $4.6 \%$, respectively. HOMA-IR, homoeostatic model assessment for $\beta$-cell function (HOMA-B) and the quantitative insulin sensitivity check index (QUICKI) were calculated according to suggested formulas ${ }^{(26)}$. Plasma total antioxidant capacity (TAC) using the ferric reducing antioxidant power method developed by Benzie \& Strain ${ }^{(27)}$, GSH by the method of Beutler \& Gelbart ${ }^{(28)}$ and malondialdehyde (MDA) values using the thiobarbituric acid-reactive substance method $^{(29)}$ were evaluated. All inter- and intra-assay CV for NO, TAC, GSH and MDA were $<5 \%$. Measurements of lipid concentrations, insulin, biomarkers of inflammation and oxidative stress were performed in a blinded fashion, in duplicate, in pairs (pre-intervention/post-intervention) at the same time.

\section{Statistical methods and sample size}

Normal distribution of variables was assessed by histogram and the Kolmogorov-Smirnov test. The analyses were conducted in all randomised subjects according to the intention-to-treat (ITT) principle. Missing values were treated based on lastobservation-carried-forward method (LOCF) ${ }^{(30)}$. LOCF ignores whether the participant's condition was improving or deteriorating at the time of dropout and instead freezes outcomes at the value observed before dropout (i.e. last observation). To establish changes in the general characteristics and daily dietary macronutrient and micronutrient intakes between the two groups, we used independent samples Student's $t$ test. Pearson's $\chi^{2}$ test was used for comparison of categorical variables. To evaluate the effects of synbiotic administration on insulin metabolism, lipid concentrations, biomarkers of inflammation and oxidative stress, we used one-way repeated measures ANOVA. The paired-samples $t$ test was used to detect within-group differences. To assess confounders, we adjusted all analyses for baseline values, age and baseline BMI with the ANCOVA test. These analyses were also performed using ANCOVA. $P<0.05$ was considered statistically significant. All statistical analyses were conducted using the Statistical Package for Social Science version 17 (SPSS Inc.).

To calculate the sample size, we used the standard formula suggested for parallel clinical trials by considering type one error $(\alpha)$ of 0.05 and type two error $(\beta)$ of 0.20 (power $=80 \%$ ). We did not find a similar study about synbiotic supplementation in RA patients for determining the sample size based on main outcome (hs-CRP); therefore, the sample size was calculated based on synbiotic supplementation in pregnant women. Based on a previous study ${ }^{(31)}$, we used an SD of $1581.6 \mathrm{ng} / \mathrm{ml}$ and a difference in mean of $13100 \mathrm{ng} / \mathrm{ml}$, considering hs-CRP as the key variable. Based on this, we needed twenty-three persons in each group. Assuming 20\% dropouts in each group, the final sample size was determined to be twenty-seven persons per group. Hs-CRP was used to estimate sample size because it is the most important variable in patients with RA. Furthermore, the largest sample size was obtained when we used this variable.

\section{Results}

At first, we invited sixty-five participants with RA; however, eleven subjects were excluded from the study as they did not meet the inclusion criteria (Fig. 1). During the intervention phase of the study, two patients were excluded in both the groups (withdrawn because of personal reasons ( $n$ 2)). However, as the analysis was conducted based on the ITT principle, all fifty-four patients with RA were included in the final analysis. On average, the rate of compliance in this study was high, such that $>90 \%$ of capsules were consumed throughout the study in both groups. No side effects were reported following intake of the synbiotic in RA patients throughout the study.

Distributions of sex, mean duration of RA, age, height, weight and BMI at baseline and after the 8-week intervention of the participants were not significantly different between the synbiotic and placebo groups (Table 1).

Comparison of dietary intakes of the study participants throughout the study revealed no significant changes in macronutrient and micronutrient intakes between the two groups (Table 2).

After the 8-week intervention, compared with the placebo, synbiotic supplementation resulted in a significant reduction in serum hs-CRP levels ( -1427.8 (SD 3267.2) $v$. +2833.4 (sD 5639.7$) \mathrm{ng} / \mathrm{ml}, P=0.001$ ). In addition, compared with the placebo, synbiotic supplementation improved DAS-28 (-1.6 (SD 0.8) $v \cdot-0.3$ (SD 0.5), $P<0.001)$ and VAS pain $(-30.4$ (SD 18.7) $v$. -11.5 (sD 15.9), $P<0.001$ ) (Table 3 ). In addition, a significant elevation in plasma NO levels $(+0.8$ (SD 4.4) $v . \quad-2.6$ (sD 4.5) $\mu \mathrm{mol} / 1, P=0.008$ ), and significant reductions in insulin values $(-13.8$ (sD 26.4) v. $+4 \cdot 2$ (sD 28.2) $\mathrm{pmol} / 1, \quad P=0 \cdot 01)$, HOMA-IR ( -0.5 (SD 1.0) $v .+0 \cdot 1(\mathrm{SD} 1 \cdot 1), P=0 \cdot 03)$ and HOMA-B (-9.4 (SD 17.9) $v .+3.3$ (SD 18.9), $P=0.01$ ) were observed following supplementation with synbiotic compared with those following the placebo. Compared with the placebo, synbiotic supplementation also resulted a significant increase in plasma GSH (+36.6 (sD 63.5) $v$. -58.5 (sD 154.4) $\mu \mathrm{mol} / \mathrm{l}, \quad P=0.005)$. Patients who received the synbiotic experienced borderline statistically significant improvement in plasma MDA $(P=0.07)$ compared with the placebo. We did not observe any significant effect on other glucose homoeostasis parameters, lipid profiles and other biomarkers of oxidative stress after synbiotic administration.

Baseline levels of plasma NO $(P<0.001)$ and DAS-28 $(P=0.004)$ were significantly different between the two groups. Therefore, we adjusted the analyses for the baseline values of biochemical parameters, age and baseline BMI. When we adjusted the analysis for baseline values of biochemical parameters, age and baseline BMI, plasma NO levels $(P=0 \cdot 17)$ 
Fig. 1. Summary of patient flow.

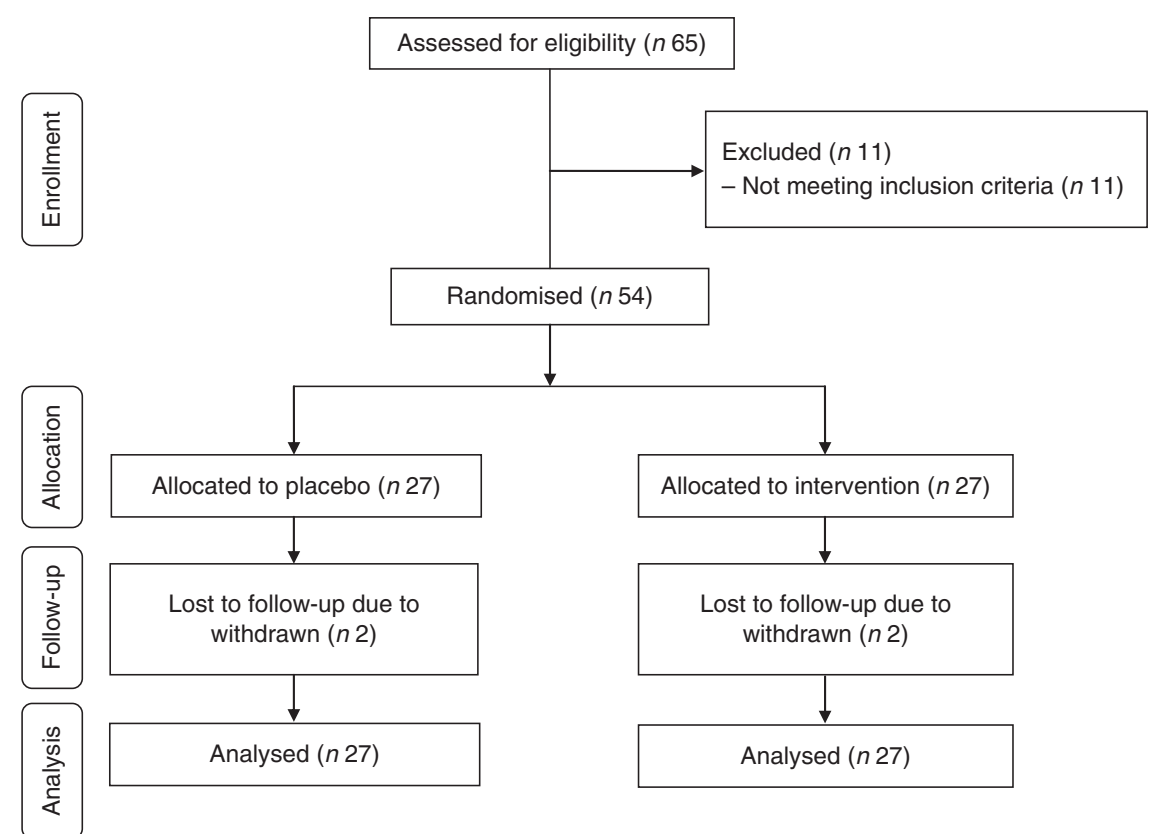

Table 1. General characteristics of study participants

(Mean values and standard deviations; numbers and percentages)

\begin{tabular}{|c|c|c|c|c|c|}
\hline & \multicolumn{2}{|c|}{$\begin{array}{l}\text { Placebo group } \\
(n 27) \\
\end{array}$} & \multicolumn{2}{|c|}{$\begin{array}{c}\text { Synbiotic group } \\
(n 27) \\
\end{array}$} & \multirow[b]{2}{*}{$P^{*}$} \\
\hline & Mean & SD & Mean & SD & \\
\hline \multicolumn{6}{|l|}{ Sex (\%) } \\
\hline Male & \multirow{2}{*}{\multicolumn{2}{|c|}{3}} & & & \multirow[t]{3}{*}{0.44} \\
\hline$n$ & & & \multirow{2}{*}{\multicolumn{2}{|c|}{$\begin{array}{c}5 \\
18.5\end{array}$}} & \\
\hline$\%$ & \multicolumn{2}{|c|}{$11 \cdot 1$} & & & \\
\hline \multicolumn{6}{|l|}{ Female } \\
\hline$n$ & \multirow{2}{*}{\multicolumn{2}{|c|}{$\begin{array}{c}24 \\
88 \cdot 9\end{array}$}} & \multirow{2}{*}{\multicolumn{2}{|c|}{$\begin{array}{c}22 \\
81.5\end{array}$}} & \\
\hline$\%$ & & & & & \\
\hline Duration of RA (years) & $7 \cdot 5$ & $6 \cdot 4$ & $7 \cdot 7$ & $6 \cdot 1$ & 0.88 \\
\hline Age (years) & 49.5 & $12 \cdot 9$ & $49 \cdot 3$ & $11 \cdot 0$ & 0.96 \\
\hline Height $(\mathrm{cm})$ & $158 \cdot 3$ & 8.9 & $162 \cdot 8$ & $8 \cdot 8$ & 0.06 \\
\hline Weight at study baseline $(\mathrm{kg})$ & 72.5 & $15 \cdot 9$ & $76 \cdot 7$ & $13 \cdot 6$ & 0.30 \\
\hline Weight at end-of-trial $(\mathrm{kg})$ & $73 \cdot 0$ & $15 \cdot 9$ & $76 \cdot 9$ & $13 \cdot 3$ & 0.32 \\
\hline Weight change (kg) & 0.4 & 1.8 & $0 \cdot 1$ & $2 \cdot 3$ & 0.60 \\
\hline BMI at study baseline $\left(\mathrm{kg} / \mathrm{m}^{2}\right)$ & $29 \cdot 1$ & $6 \cdot 7$ & $29 \cdot 2$ & 5.9 & 0.93 \\
\hline BMI at end-of-trial $\left(\mathrm{kg} / \mathrm{m}^{2}\right)$ & $29 \cdot 2$ & $6 \cdot 7$ & $29 \cdot 2$ & $5 \cdot 8$ & 0.99 \\
\hline BMI change $\left(\mathrm{kg} / \mathrm{m}^{2}\right)$ & 0.1 & 0.7 & 0.04 & $0 \cdot 8$ & 0.52 \\
\hline \multicolumn{6}{|l|}{ Current medication } \\
\hline Methotrexate & \multirow{2}{*}{\multicolumn{2}{|c|}{26}} & & & \multirow[t]{3}{*}{$1.00 \dagger$} \\
\hline$n$ & & & \multirow{2}{*}{\multicolumn{2}{|c|}{$\begin{array}{c}26 \\
96 \cdot 3\end{array}$}} & \\
\hline$\%$ & \multicolumn{2}{|c|}{$96 \cdot 3$} & & & \\
\hline Prednisolone & \multirow{2}{*}{\multicolumn{2}{|c|}{25}} & & & \multirow[t]{3}{*}{$0.63 \dagger$} \\
\hline$n$ & & & \multirow{2}{*}{\multicolumn{2}{|c|}{$\begin{array}{c}24 \\
88.9\end{array}$}} & \\
\hline$\%$ & \multicolumn{2}{|c|}{$92 \cdot 6$} & & & \\
\hline Hydroxychloroquine & \multirow{3}{*}{\multicolumn{2}{|c|}{$\begin{array}{c}18 \\
66 \cdot 7\end{array}$}} & & & $0.77 \dagger$ \\
\hline$n$ & & & \multirow{2}{*}{\multicolumn{2}{|c|}{$\begin{array}{c}19 \\
70.4\end{array}$}} & \\
\hline$\%$ & & & & & \\
\hline
\end{tabular}

RA, rheumatoid arthritis

* Obtained from independent $t$ test.

$\dagger$ Pearson's $x^{2}$ test.

became non-significant, whereas serum LDL-cholesterol $(P=0.02)$ became statistically significant, and other findings did not alter (Table 4$)$.
Table 2. Dietary intakes of study participants throughout the study (Mean values and standard deviations)

\begin{tabular}{|c|c|c|c|c|c|}
\hline & \multicolumn{2}{|c|}{$\begin{array}{l}\text { Placebo group } \\
\quad(n 27)\end{array}$} & \multicolumn{2}{|c|}{$\begin{array}{l}\text { Synbiotic group } \\
(\text { ( } 27)\end{array}$} & \multirow[b]{2}{*}{$P^{*}$} \\
\hline & Mean & SD & Mean & SD & \\
\hline Energy (kJ/d) & 9581 & 1038 & 9749 & 1176 & 0.58 \\
\hline Energy (kcal/d) & 2290 & 248 & 2330 & 281 & 0.58 \\
\hline Carbohydrates (g/d) & 311.6 & 50.6 & 329.3 & 61.7 & 0.25 \\
\hline Protein $(\mathrm{g} / \mathrm{d})$ & 84.9 & $15 \cdot 1$ & $85 \cdot 2$ & $18 \cdot 6$ & 0.94 \\
\hline Fat $(g / d)$ & 81.6 & $10 \cdot 1$ & 78.6 & $16 \cdot 6$ & 0.41 \\
\hline $\operatorname{SFA}(\mathrm{g} / \mathrm{d})$ & 24.7 & 4.6 & 24.5 & 5.6 & 0.90 \\
\hline PUFA (g/d) & $25 \cdot 9$ & $5 \cdot 5$ & $24 \cdot 8$ & $6 \cdot 8$ & 0.50 \\
\hline MUFA $(g / d)$ & 22.5 & $5 \cdot 3$ & 21.6 & 6.6 & 0.59 \\
\hline Cholesterol $(\mathrm{mg} / \mathrm{d})$ & 233.6 & $126 \cdot 9$ & 193.6 & 108.4 & 0.21 \\
\hline $\operatorname{TDF}(\mathrm{g} / \mathrm{d})$ & $18 \cdot 3$ & $5 \cdot 3$ & $19 \cdot 8$ & 4.7 & 0.29 \\
\hline
\end{tabular}

\section{Discussion}

In this research, we assessed the effects of synbiotic supplementation on clinical and metabolic parameters in patients with RA. We found that synbiotic supplementation for 8 weeks among patients with RA had beneficial effects on hs-CRP, DAS-28, VAS, NO, insulin levels, HOMA-IR, HOMA-B and GSH levels; however, it did not affect other glucose homoeostasis parameters, lipid profiles and other biomarkers of oxidative stress. However, when the analysis was conducted without the ITT approach, no significant changes were seen in our findings.

In unadjusted analyses, the current study indicated that synbiotic supplementation for 8 weeks among subjects with RA had beneficial effects on serum hs-CRP, DAS-28 and plasma NO levels compared with the placebo. When we adjusted the analyses for baseline values of biochemical variables, age and 


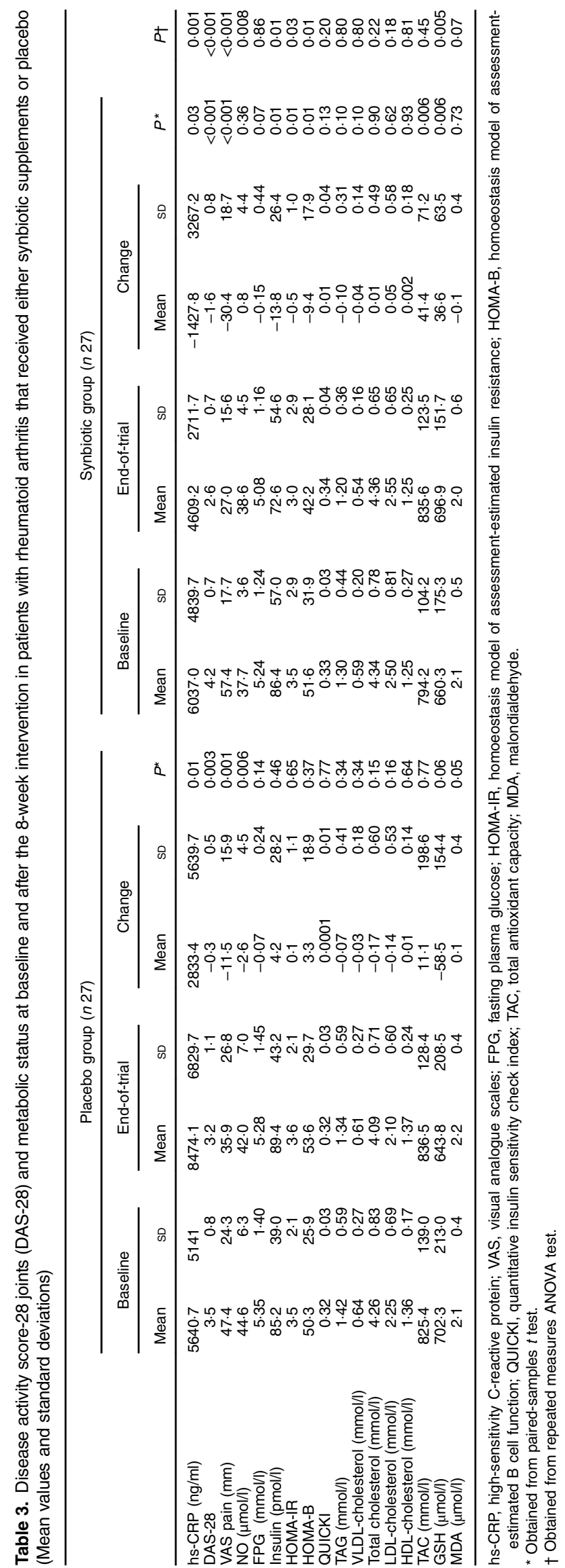

baseline BMI, the change in plasma NO levels was not significantly different between the groups. Data on the effects of synbiotic supplementation in RA subjects are limited. We have previously shown that probiotic intake for 8 weeks among RA subjects improved DAS-28 and serum hs-CRP values, but did not influence plasma NO levels ${ }^{(10)}$. In addition, supporting our findings, supplementation with $L$. case $i$ among patients with RA for 8 weeks resulted in a significant decrease in VAS ${ }^{(23)}$. In an animal study, B. coagulans plus inulin significantly improved inflammatory factors and clinical parameters in induced $\mathrm{RA}^{(3)}$. Furthermore, few animal studies reported that probiotics including Lactobacillus $G G$ and $L$. casei had anti-RA effects ${ }^{(32,33)}$. In another study, supplementation with synbiotics in adults with non-alcoholic fatty liver disease over 28 weeks reduced inflammatory factors ${ }^{(34)}$. However, the intake of synbiotic supplements did not affect serum CRP levels among men with a low serum enterolactone concentration after 6 weeks ${ }^{(35)}$. Pineda et $a l .{ }^{(36)}$ observed that probiotic supplementation among patients with active RA for $30 \mathrm{~d}$ had no significant effect on inflammatory cytokines and the Health Assessment Questionnaire. The exact mechanisms of the beneficial effects of synbiotics on clinical symptoms and inflammatory factors in RA patients are unknown. The up-regulation of IL-18 protein expression produced by SCFA ${ }^{(37)}$ and increased production of the methylketone family in the gut following supplementation with synbiotics ${ }^{(38)}$ might result in its anti-inflammatory effects. SCFA may lower serum hs-CRP levels through blocking the enzymatic synthesis of hepatic CRP. CRP is synthesised by the liver in response to releasing factors by fat cells such as IL-6 ${ }^{(39)}$. In a study by Hegazy \& El-Bedewy ${ }^{(40)}$ it was observed that the consumption of probiotics by patients with ulcerative colitis for 8 weeks significantly ameliorated the inflammation by decreasing concentrations of IL-6, expression of TNF- $\alpha$ and $\mathrm{NF}-\kappa \mathrm{B}$. Likely, decreasing concentrations of IL- 6 indirectly cause a decreasing production of CRP.

In unadjusted analyses, we found that synbiotic administration for 8 weeks in patients with RA decreased serum insulin concentrations, HOMA-IR and HOMA-B compared with the placebo, whereas it did not affect FPG, QUICKI and lipid concentrations. When we adjusted the analyses for baseline values of biochemical variables, age and baseline BMI, a significant change in serum total cholesterol was observed. Some researchers have documented the beneficial effects of synbiotic supplementation on insulin metabolism and lipid profiles among patients without RA in former studies. We have previously demonstrated that synbiotic administration for 6 weeks among subjects with GDM had favourable effects on insulin metabolism, TAG and VLDL-cholesterol concentrations, but did not affect FPG and other lipid fractions ${ }^{(22)}$. Moreover, insulin resistance was significantly improved following the intake of synbiotics among subjects with the metabolic syndrome after 28 weeks of treatment, but unchanged lipid profiles $^{(17)}$. The same findings were seen by others after the consumption of synbiotic supplements among patients with non-alcoholic steatohepatitis for 24 weeks $^{(41)}$ and in highfructose-fed rats ${ }^{(42)}$. However, Schaafsma et al. ${ }^{(43)}$ found that synbiotic administration in male volunteers for 3 weeks significantly reduced the total-, LDL- and LDL-/HDL-cholesterol, 
Table 4. Adjusted changes in metabolic variables in patients with rheumatoid arthritis that received either synbiotic supplements or placebo (Mean values with their standard errors)

\begin{tabular}{|c|c|c|c|c|c|}
\hline & \multicolumn{2}{|c|}{ Placebo group ( $n$ 27) } & \multicolumn{2}{|c|}{ Synbiotic group ( $n$ 27) } & \multirow[b]{2}{*}{$P^{*}$} \\
\hline & Mean & SE & Mean & SE & \\
\hline hs-CRP (ng/ml) & $2762 \cdot 5$ & $816 \cdot 8$ & $-1356 \cdot 9$ & $816 \cdot 8$ & 0.001 \\
\hline DAS-28 & -0.4 & 0.1 & -1.4 & 0.1 & $<0.001$ \\
\hline VAS pain (mm) & $-12 \cdot 9$ & 3.2 & -28.9 & 3.2 & 0.001 \\
\hline $\mathrm{NO}(\mu \mathrm{mol} / \mathrm{l})$ & -1.9 & 0.9 & 0.1 & 0.9 & 0.17 \\
\hline $\mathrm{FPG}(\mathrm{mmol} / \mathrm{l})$ & -0.07 & 0.06 & -0.15 & 0.06 & 0.38 \\
\hline Insulin (pmol/l) & $4 \cdot 2$ & 5.4 & $-13 \cdot 8$ & $5 \cdot 4$ & 0.01 \\
\hline HOMA-IR & 0.1 & 0.2 & -0.5 & 0.2 & 0.03 \\
\hline HOMA-B & $3 \cdot 2$ & 3.4 & $-9 \cdot 3$ & 3.4 & 0.01 \\
\hline QUICKI & -0.001 & 0.007 & 0.01 & 0.007 & 0.16 \\
\hline TAG $(\mathrm{mmol} / \mathrm{l})$ & -0.06 & 0.06 & -0.11 & 0.06 & 0.49 \\
\hline VLDL-cholesterol (mmol/l) & -0.02 & 0.02 & -0.05 & 0.02 & 0.49 \\
\hline Total cholesterol $(\mathrm{mmol} / \mathrm{l})$ & -0.18 & 0.09 & 0.02 & 0.09 & 0.10 \\
\hline LDL-cholesterol (mmol/l) & -0.20 & 0.09 & $0 \cdot 10$ & 0.09 & 0.02 \\
\hline HDL-cholesterol (mmol/I) & 0.02 & 0.03 & -0.005 & 0.03 & 0.56 \\
\hline TAC $(\mathrm{mmol} / \mathrm{l})$ & $22 \cdot 2$ & $24 \cdot 0$ & $30 \cdot 3$ & $24 \cdot 0$ & 0.81 \\
\hline $\mathrm{GSH}(\mu \mathrm{mol} / \mathrm{l})$ & $-53 \cdot 3$ & $21 \cdot 0$ & $31 \cdot 3$ & $21 \cdot 0$ & 0.007 \\
\hline MDA ( $\mu \mathrm{mol} / \mathrm{l})$ & 0.2 & 0.1 & -0.02 & 0.1 & 0.07 \\
\hline
\end{tabular}

hs-CRP, high-sensitivity C-reactive protein; DAS-28, disease activity score-28 joints; VAS, visual analogue scales; FPG, fasting plasma glucose; HOMA-IR, homoeostasis model of assessment-estimated insulin resistance; HOMA-B, homoeostasis model of assessment-estimated B cell function; QUICKI, quantitative insulin sensitivity check index; TAC, total antioxidant capacity; MDA, malondialdehyde.

* Obtained from ANCOVA adjusted for baseline values + age and baseline BMI.

whereas it did not affect TAG levels. The absence of beneficial effects of synbiotics on lipid profiles in our study compared with that in other studies may be mediated by different study designs, different dosages of used probiotic and inulin, types and quality of used probiotic bacteria and inulin as well as duration of the intervention. Prior studies have reported that insulin resistance ${ }^{(44,45)}$ and oxidative stress ${ }^{(46)}$, independently, may impair disease activity in subjects with RA. Therefore, synbiotics, because of their improving effects on insulin metabolism, anti-inflammatory and anti-oxidative actions, may be useful to decrease complications in subjects with RA. Synbiotics might improve insulin metabolism through the modification of gut flora and elevation of faecal $\mathrm{pH}^{(18)}$, decreased production of pro-inflammatory cytokines ${ }^{(19)}$ and modulating NF- $\kappa \mathrm{B}^{(47)}$.

This study demonstrated that taking synbiotic supplements for 8 weeks among subjects with RA significantly increased plasma GSH levels, but did not affect other biomarkers of oxidative stress compared with the placebo. In agreement with our findings, no significant effect on MDA, TAC, superoxide dismutase, glutathione peroxidase, and catalase activities following supplementation with $L$. casei among patients with RA for 8 weeks was observed ${ }^{(48)}$. Probiotic intake for 12 weeks among pregnant women increased plasma GSH and TAC, but did not affect MDA values ${ }^{(49)}$. Likewise, we have previously shown that synbiotic food consumption for 9 weeks among pregnant women resulted in a significant elevation in plasma GSH concentrations ${ }^{(50)}$. Probiotic administration among subjects with major depressive disorder for 8 weeks increased GSH values, but did not influence plasma TAC values ${ }^{(51)}$. Synbiotic intake may decrease oxidative stress through improved inflammatory factors resulting from the production of SCFA in the colon ${ }^{(52)}$, increased generation of $\mathrm{NO}^{(53)}$, and its impact on decreased biomarkers including oxidised LDL, 8-isoprostanes and GSH ratio ${ }^{(54)}$.

This research had some limitations. Because of funding limitations, we did not assess the compliance through quantification of faecal-bacteria loads and SCFA. Moreover, further studies are needed to evaluate gene expression related to inflammatory markers and insulin to explore the plausible mechanism and confirm our findings.

Overall, our study demonstrated that synbiotic supplementation for 8 weeks among patients with RA had beneficial effects on hs-CRP, DAS-28, VAS, NO, insulin levels, HOMA-IR, HOMA-B and GSH levels; however, it did not affect other glucose homoeostasis parameters, lipid profiles and other biomarkers of oxidative stress.

\section{Acknowledgements}

The present study was supported by a grant from the Vicechancellor for Research, KUMS and Iran.

Z. A. contributed in conception, design, statistical analysis and drafting of the manuscript. B. Z., S. F., H. R. G. and F. B. contributed to data collection and manuscript drafting. The final version was confirmed by all authors for submission.

None of the authors has any conflicts of interest to declare.

\section{References}

1. Aletaha D, Martinez-Avila J, Kvien TK, et al. (2012) Definition of treatment response in rheumatoid arthritis based on the simplified and the clinical disease activity index. Ann Rheum Dis 71, 1190-1196.

2. Diamanti AP, Manuela Rosado M, Lagana B, et al. (2016) Microbiota and chronic inflammatory arthritis: an interwoven link. J Transl Med 14, 233. 
3. Abhari K, Shekarforoush SS, Hosseinzadeh S, et al. (2016) The effects of orally administered Bacillus coagulans and inulin on prevention and progression of rheumatoid arthritis in rats. Food Nutr Res 60, 30876

4. Giles JT, Danielides S, Szklo M, et al. (2015) Insulin resistance in rheumatoid arthritis: disease-related indicators and associations with the presence and progression of subclinical atherosclerosis. Arthritis Rheumatol 67, 626-636.

5. Maruotti N, d'Onofrio F \& Cantatore FP (2015) Metabolic syndrome and chronic arthritis: effects of anti-TNF-alpha therapy. Clin Exp Med 15, 433-438.

6. Bradham WS, Ormseth MJ, Oeser A, et al. (2014) Insulin resistance is associated with increased concentrations of NT-proBNP in rheumatoid arthritis: IL-6 as a potential mediator. Inflammation 37, 801-808.

7. Geronikaki AA \& Gavalas AM (2006) Antioxidants and inflammatory disease: synthetic and natural antioxidants with anti-inflammatory activity. Comb Chem High Throughput Screen 9, 425-442.

8. Avina-Zubieta JA, Choi HK, Sadatsafavi M, et al. (2008) Risk of cardiovascular mortality in patients with rheumatoid arthritis: a meta-analysis of observational studies. Arthritis Rheum 59 1690-1697.

9. Su CC, Chen Ie C, Young FN, et al. (2013) Risk of diabetes in patients with rheumatoid arthritis: a 12-year retrospective cohort study. J Rheumatol 40, 1513-1518.

10. Zamani B, Golkar HR, Farshbaf S, et al. (2016) Clinical and metabolic response to probiotic supplementation in patients with rheumatoid arthritis: a randomized, double-blind, placebo-controlled trial. Int J Rheum Dis 19, 869-879.

11. Kano H, Kaneko T \& Kaminogawa S (2002) Oral intake of Lactobacillus delbrueckii subsp. bulgaricus OLL1073R-1 prevents collagen-induced arthritis in mice. J Food Prot 65, 153-160.

12. Strowski MZ \& Wiedenmann B (2009) Probiotic carbohydrates reduce intestinal permeability and inflammation in metabolic diseases. Gut 58, 1044-1045.

13. de Vrese M \& Schrezenmeir J (2008) Probiotics, prebiotics, and synbiotics. Adv Biochem Eng Biotechnol 111, 1-66.

14. Frece J, Kos B, Svetec IK, et al. (2009) Synbiotic effect of Lactobacillus helveticus M92 and prebiotics on the intestinal microflora and immune system of mice. J Dairy Res 76, 98-104.

15. Worthley DL, Le Leu RK, Whitehall VL, et al. (2009) A human, double-blind, placebo-controlled, crossover trial of prebiotic, probiotic, and synbiotic supplementation: effects on luminal, inflammatory, epigenetic, and epithelial biomarkers of colorectal cancer. Am J Clin Nutr 90, 578-586.

16. Akram KA, Tofighiyan $\mathrm{T} \&$ Rakhshani MH (2015) Effects of synbiotics on inflammatory markers in patients with type 2 diabetes mellitus. Glob J Health Sci 7, 1-5.

17. Eslamparast T, Zamani F, Hekmatdoost A, et al. (2014) Effects of synbiotic supplementation on insulin resistance in subjects with the metabolic syndrome: a randomised, double-blind, placebo-controlled pilot study. Br J Nutr 112, 438-445.

18. Compare D, Coccoli P, Rocco A, et al. (2012) Gut-liver axis: the impact of gut microbiota on non alcoholic fatty liver disease. Nutr Metab Cardiovasc Dis 22, 471-476.

19. Li Z, Yang S, Lin H, et al. (2003) Probiotics and antibodies to TNF inhibit inflammatory activity and improve nonalcoholic fatty liver disease. Hepatology 37, 343-350.

20. Reneses S, Pestana L \& Garcia A (2012) Comparison of the 1987 ACR criteria and the 2010 ACR/EULAR criteria in an inception cohort of patients with recent-onset inflammatory polyarthritis. Clin Exp Rheumatol 30, 417-420.

21. Pereira DI, McCartney AL \& Gibson GR (2003) An in vitro study of the probiotic potential of a bile-salt-hydrolyzing Lactobacillus fermentum strain, and determination of its cholesterol-lowering properties. Appl Environ Microbiol 69 , 4743-4752.

22. Ahmadi S, Jamilian M, Tajabadi-Ebrahimi M, et al. (2016) The effects of synbiotic supplementation on markers of insulin metabolism and lipid profiles in gestational diabetes: a randomised, double-blind, placebo-controlled trial. $\mathrm{Br} J \mathrm{Nutr}$ 116, 1394-1401.

23. Vaghef-Mehrabany E, Alipour B, Homayouni-Rad A, et al. (2014) Probiotic supplementation improves inflammatory status in patients with rheumatoid arthritis. Nutrition 30, 430-435.

24. Ainsworth BE, Haskell WL, Whitt MC, et al. (2000) Compendium of physical activities: an update of activity codes and MET intensities. Med Sci Sports Exerc 32, S498-S504.

25. Tatsch E, Bochi GV, Pereira Rda S, et al. (2011) A simple and inexpensive automated technique for measurement of serum nitrite/nitrate. Clin Biochem 44, 348-350.

26. Pisprasert V, Ingram KH, Lopez-Davila MF, et al. (2013) Limitations in the use of indices using glucose and insulin levels to predict insulin sensitivity: impact of race and gender and superiority of the indices derived from oral glucose tolerance test in African Americans. Diabetes Care 36, 845-853.

27. Benzie IF \& Strain JJ (1996) The ferric reducing ability of plasma (FRAP) as a measure of 'antioxidant power': the FRAP assay. Anal Biochem 239, 70-76.

28. Beutler E \& Gelbart T (1985) Plasma glutathione in health and in patients with malignant disease. J Lab Clin Med 105, 581-584.

29. Janero DR (1990) Malondialdehyde and thiobarbituric acid-reactivity as diagnostic indices of lipid peroxidation and peroxidative tissue injury. Free Radic Biol Med 9, 515-540.

30. Lachin JM (2016) Fallacies of last observation carried forward analyses. Clin Trials 13, 161-168.

31. Taghizadeh $M$ \& Asemi $Z$ (2014) Effects of synbiotic food consumption on glycemic status and serum hs-CRP in pregnant women: a randomized controlled clinical trial. Hormones (Athens) 13, 398-406.

32. Baharav E, Mor F, Halpern M, et al. (2004) Lactobacillus GG bacteria ameliorate arthritis in Lewis rats. J Nutr 134, 1964-1969.

33. So JS, Kwon HK, Lee CG, et al. (2008) Lactobacillus casei suppresses experimental arthritis by down-regulating $\mathrm{T}$ helper 1 effector functions. Mol Immunol 45, 2690-2699.

34. Eslamparast T, Poustchi H, Zamani F, et al. (2014) Synbiotic supplementation in nonalcoholic fatty liver disease: a randomized, double-blind, placebo-controlled pilot study. Am J Clin Nutr 99, 535-542.

35. Holma R, Kekkonen RA, Hatakka K, et al. (2014) Low serum enterolactone concentration is associated with low colonic Lactobacillus-Enterococcus counts in men but is not affected by a synbiotic mixture in a randomised, placebo-controlled, doubleblind, cross-over intervention study. Br J Nutr 111, 301-309.

36. Pineda Mde L, Thompson SF, Summers K, et al. (2011) A randomized, double-blinded, placebo-controlled pilot study of probiotics in active rheumatoid arthritis. Med Sci Monit 17, CR347-CR354.

37. Kalina U, Koyama N, Hosoda T, et al. (2002) Enhanced production of IL-18 in butyrate-treated intestinal epithelium by stimulation of the proximal promoter region. Eur J Immunol 32, 2635-2643.

38. Vitali B, Ndagijimana M, Cruciani F, et al. (2010) Impact of a synbiotic food on the gut microbial ecology and metabolic profiles. BMC Microbiol 10, 4.

39. Kinoshita A, Onoda H, Imai N, et al. (2015) C-reactive protein as a prognostic marker in patients with hepatocellular carcinoma. Hepatogastroenterology 62, 966-970.

40. Hegazy SK \& El-Bedewy MM (2010) Effect of probiotics on pro-inflammatory cytokines and NF-kappaB activation in ulcerative colitis. World J Gastroenterol 16, 4145-4151. 
41. Malaguarnera M, Vacante M, Antic T, et al. (2012) Bifidobacterium longum with fructo-oligosaccharides in patients with non alcoholic steatohepatitis. Dig Dis Sci 57, 545-553.

42. Yadav H, Jain S \& Sinha PR (2007) Antidiabetic effect of probiotic dahi containing Lactobacillus acidophilus and Lactobacillus casei in high fructose fed rats. Nutrition 23, 62-68.

43. Schaafsma G, Meuling WJ, van Dokkum W, et al. (1998) Effects of a milk product, fermented by Lactobacillus acidophilus and with fructo-oligosaccharides added, on blood lipids in male volunteers. Eur J Clin Nutr 52, 436-440.

44. Chung CP, Oeser A, Solus JF, et al. (2008) Inflammationassociated insulin resistance: differential effects in rheumatoid arthritis and systemic lupus erythematosus define potential mechanisms. Arthritis Rheum 58, 2105-2112.

45. Dessein PH \& Joffe BI (2006) Insulin resistance and impaired beta cell function in rheumatoid arthritis. Arthritis Rheum $\mathbf{5 4}$, $2765-2775$.

46. Nakajima A, Aoki Y, Shibata Y, et al. (2014) Identification of clinical parameters associated with serum oxidative stress in patients with rheumatoid arthritis. Mod Rheumatol 24, 926-930.

47. Grimoud J, Durand H, de Souza S, et al. (2010) In vitro screening of probiotics and synbiotics according to anti-inflammatory and anti-proliferative effects. Int J Food Microbiol 144, 42-50.

48. Vaghef-Mehrabany E, Homayouni-Rad A, Alipour B, et al. (2016) Effects of probiotic supplementation on oxidative stress indices in women with rheumatoid arthritis: a randomized double-blind clinical trial. J Am Coll Nutr 35, 291-299.

49. Jamilian M, Bahmani F, Vahedpoor Z, et al. (2016) Effects of probiotic supplementation on metabolic status in pregnant women: a randomized, double-blind, placebo-controlled trial. Arch Iran Med 19, 687-682.

50. Taghizadeh M, Hashemi T, Shakeri H, et al. (2014) Synbiotic food consumption reduces levels of triacylglycerols and VLDL, but not cholesterol, LDL, or HDL in plasma from pregnant women. Lipids 49, 155-161.

51. Akkasheh G, Kashani-Poor Z, Tajadadi-Ebrahimi $\mathrm{M}$, et al. (2016) Clinical and metabolic response to probiotic administration in patients with major depressive disorder: a randomized, double-blind, placebo-controlled trial. Nutrition 32, 315-320.

52. Sadrzadeh-Yeganeh H, Elmadfa I, Djazayery A, et al. (2010) The effects of probiotic and conventional yoghurt on lipid profile in women. Br J Nutr 103, 1778-1783.

53. Sobko T, Huang L, Midtvedt T, et al. (2006) Generation of NO by probiotic bacteria in the gastrointestinal tract. Free Radic Biol Med 41, 985-991.

54. Kullisaar T, Songisepp E, Mikelsaar M, et al. (2003) Antioxidative probiotic fermented goats' milk decreases oxidative stress-mediated atherogenicity in human subjects. Br J Nutr 90, 449-456. 